\title{
La représentation des femmes dans la littérature francophone
}

\author{
Martha MZITE \\ Département de Langues Étrangères, Université du Zimbabwe - Zimbabwe
}

Reçu le 21 mai 2019 | Acceptéle 30 juin 2019

\begin{abstract}
RÉSUMÉ. Cet article cherche à contribuer au discours littéraire francophone en effectuant une analyse comparative des représentations de la peinture des inégalités sociales dans la trilogie de Keita Fatoumata. Les trois romans illustrent de manière concourante ce que la femme africaine vit tous les jours et ils mettent également en lumière la façon dont la femme est subjuguée par les habitudes coutumières et culturelles en Afrique postcoloniale. La question de recherche principale s'articule autour du vécu global des femmes marginalisées. S'inspirant des théories féministes postcoloniales et de la masculinité hégémoniques, l'article examinera comment le patriarche étouffe la féminité. La théorisation de Spivak « les subalternes, peuvent-elles parler ? » est la question autour de laquelle cette communication s'articule. «La conscience de l'oppression » de Simone De Beauvoir démontre qu'on partageant leurs expériences, les femmes enchainées peuvent se détacher de toutes les maux qui les oppriment. L'analyse de ces romans montre que les questions de genre amplifient la soumission de la femme en Afrique. Cette étude aborde le lévirat, l'excision et la polygamie. Nous concluons que la femme peut se détacher des inégalités sociales qui l'étouffent.
\end{abstract}

Mots-clés : excision, femme, lévirat, patriarche, polygamie

\begin{abstract}
This article seeks to contribute to Francophone literary discourse by performing a comparative analysis of the portrayal of social inequalities in Keïta Fatoumata's trilogy. The three novels illustrate in a concise way what the African woman lives every day and they also highlight how the woman is subjugated by customary and cultural habits in postcolonial Africa. The main research question revolves around the overall experience of marginalized women. Inspired by post-colonial feminist theories and hegemonic masculinity, the article will examine how the patriarch stifles femininity. Spivak's Theorization "Can Subalterns Speak? Is the question around which this communication is articulated. Simone De Beauvoir's "conscience of oppression" demonstrates that by sharing their experiences, chained women can detach themselves from all the ills that oppress them. The analysis of these novels shows that gender issues magnify the submission of women in Africa. This study addresses levirate, excision and polygamy. The article concludes that women can be detached from the social inequalities that suffocate them.
\end{abstract}

Keywords: polygamy, excision, levirate, woman, patriarch

auteur correspondant : mzitem@africau.edu

Pour citer cet article (Style APA) : Mzite. (2019). La représentation des femmes dans la littérature africaine. Francisola: Revue Indonésienne de la langue et la littérature françaises, 4(1), 30-36 . doi: 10.17509/francisola.v4i1.20339. 


\section{INTRODUCTION}

La condition de la femme est toujours très incertaine en Afrique étant donné que la société impose des codes à travers les us et les coutumes. Naamane (1988, p.105) croit que « la femme n'a souvent que la maternité pour combler le vide de sa vie conjugale; avoir un enfant est d'ailleurs partie intégrante de son devoir d'épouse et l'on sait que ce devoir est, dans notre société fondateur $\mathrm{du}$ lien marital. La position d'épouse se renforce lors de la grossesse, de l'accouchement, et surtout lorsque l'enfant né est de sexe mâle. »Cette manière de pensée augmente les effets des inégalités sociales qui pèsent sur les femmes.

La façon dont on mène sa vie est souvent due aux croyances ou aux convictions philosophiques ou aux convictions » de la société, suivant la pensée de Zénith (2000, p.119). L'analyse de notre corpus illustre que la présence de la tradition et des mœurs anciennes en Afrique soutiennent le rapport entre les femmes et les hommes. À propos des écarts dans la société africaine, on est témoin à une croyance ancienne où la femme fait partie des possessions de son mari, ainsi, elle est ensuite confinée à une position minimale de l'échelle sociale. À travers la gamme de protagonistes dans les romans, le lecteur partage le quotidien de ces femmes dont les ailes ont été coupées par la société au nom de la patriarchie. Cette communication, accentue la contribution important des romanciers dans leurs combats pour accentuer le besoin de faire sortir les femmes de leur marginalisation. L'objectif de cette étude est d'étudier la condition de la femme africaine pour témoigner les altérités qui se présentent entre elles et les hommes.

Cet article est structuré en plusieurs sections. Chaque partie est consacrée à l'analyse de la tyrannie des us et des coutumes qui asservissement les femmes africaines. Même si la situation de la femme n'est pas la même partout en Afrique, la romancière se permet à reconnaître le statut inférieur donné à la femme, de ce fait, elle devient une mineure perpétuelle dans la société. Cette situation est le résultat du système ancestral qui ordonne à la femme les vices comme l'asservissement. Cependant, la femme africaine ne reste pas silencieuse face à cette situation, elle se bat tant qu'elle le peut pour se protéger et assurer sa vie. Le corpus augmenté par d'autres romans permet d'exposer plusieurs opinions. De ce fait, d'autres romans comme corpus secondaire seront consultés.

\section{MÉTHODE}

Cette étude vise à analyser l'image du statut de la femme africaine dans la trilogie de Fatoumata Keïta: Sous fer (2013), Quand les cauris se taisent (2017) et Les mamelles de l'amour (2017). Les objectifs de cette étude sont les suivants : Établir comment la femme est assujettie par la société patriarcale et exposer la réponse de la femme face aux coutumes et aux habitudes de la communauté patriarche. Pour y parvenir, cette étude propose l'analyse thématique et l'analyse des anaphores pour accentuer la thématique. L'analyse de données a été inspirée par la théorie de Simone de Beauvoir de la conscience de l'oppression et celle de Spivak qui se pose la question suivante : « Les subalternes peuvent-elles parler ? » cité par Chassain et al (2016:8).

La vision de Spivak offre une perspective marxiste et féministe aussi. La question précédente renvoie à la capacité que la femme possède. La capacité de se sauver des subjugations patriarcales. Le subalterne est le " paysan illettré. La femme du Tiers monde ... » selon Bardolph (2002, p.41). La théorie de Simone de Beauvoir démontre la source des altérités des femmes. Beauvoir propose ce qui suit : " On ne naît pas femme, on le devient » (De Beauvoir, 1976, p.13). Elle témoigne que la distance entre l'homme et la femme se base sur les convictions culturelles et non pas sur la nature elle-même. Pour analyser le corpus, d'autres romans secondaires qui traitent les mêmes thèmes seront consultés aussi. La lecture d'articles et d'autres sources médiatiques pertinentes sera utilisée également.

\section{RÉSULTATS ET DISCUSSION}


Dans Sous fer, Kanda a épousé Fata sous le régime monogame contre la volonté de sa famille. Ce couple endure les effets de cette décision tous les jours. Dans cette optique, ils deviennent proies d'une exclusion de la part de la famille de Kanda. Ils ont une fille, Nana qu'ils élèvent dans un encadrement apparemment affranchi des pesanteurs traditionnelles. Elle vise être médecin et ses parents la protègent de l'excision. Son dilemme oscille entre les poids des coutumes et le désir de s'émanciper. Dans Quand les cauris se taisent, Nana, qui est étudiante à l'université présume déjà avoir les rênes de sa vie, cependant elle entrevue des épreuves imprévus. Le mari de Titi veut se remarier parce qu'elle ne peut pas enfanter. Dans Les Mamelles de l'amour, la mort de Kary bouleverse la vie de Nana. Son univers se démolit devant ses yeux. Son rejet de la requête d'épouser le cousin de son décédé mari catalyse l'antagonisme entre elle et sa belle-famille.

Fatoumata Keïta dans Sous fer évoque le mariage forcé. À travers Fata et sa fille Nana, la romancière dépeint les coutumes en rapport avec des mariages qui assujettissent les femmes dans certaines cultures en Afrique. Le père de Fata lui tient les propos suivants :

D'ailleurs, tu n'es pas sans savoir que tu es déjà fiancée. Mon cousin Hama, tu le sais, a attaché depuis ta naissance un fils autour de ton poignet afin de te réserver à son fils Bouba. Considère-toi donc comme déjà mariée, Fata je ne veux pas que tu me couvres de honte parmi mes semblables. (Keïta, 2013, p.12)

La romancière montre l'ironie de cette coutume où les parents réservent une épouse pour un fils comme on réserve un achat. Nana ne pouvait pas réagir autrement, mais elle s'est contentée de penser qu'un " jour viendrait où elle réussirait à briser toutes les pesanteurs sociales pour se marier avec son amoureux » Keïta (2013, p.12). À l'âge de dixhuit ans ses oncles l'ont mariée sans sa volonté. Lisons ce que Fata dit à sa fille un soir pendant la visite de ses oncles : " Ma fille ! Tes fiançailles avec Magandian ont été faites...tes oncles ont accordé ta main à Magandian. Parlant à son père Nana déclare ce qui suit : «Si la décision de mon mariage doit venir d'une personne autre que moi je serai prête à tout pour faire changer les choses » (Keïta, 2013, p.65). Même si Nana ne voulait pas épouser Magandian, elle ne pouvait pas faire autrement. La marginalisation de la femme produit un effet d'affliction, de frustration et d'isolement. Dans la citation en haut, nous avons mis en gras les termes anaphoriques infidèles liés au mariage de Nana. Les mots en gras mettent en valeur l'importance accordée à la fidélité de Nana.

Plus tard, nous voyons la cérémonie pour le paiement de la dot pour Nana dans la citation suivante : «Noble femme qui sais donner de beaux fruits, accepte ces présents. C'est de la part de Magandian qui cherche à intégrer la famille Magassouba à travers les liens sacrés du mariage qu'il veut contracter avec Nana » (Keïta, 2013, p.85) À travers cette présentation, Nana devient l'épouse de Magandian sans sa volonté.

Les mariages d'enfants, selon l'écriture, sont une violation des droits de l'enfant, surtout les droits des jeunes filles. Elles sont dépossédées de leur enfance et elles n'ont ni la sagesse ni assez de conception pour autoriser et concevoir un tel contrat. Ces mariages ont des effets néfastes sur le bien-être des jeunes filles qui ne sont pas socialement préparées. Ceci implique qu'elles ne sont pas encore prêtes pour affronter les obligations qu'entraîne un mariage. D'un autre point de vue, ces jeunes filles ne sont pas disposées à supporter une grossesse et un accouchement. De plus, beaucoup d'entre elles enfantent prématurément, mettant au monde un enfant qui aura moins de possibilités de survivance. Le processus de l'accouchement peut être difficile pour la jeune mère et son enfant.

Kesteloot (2001, p.284) croit que «le mariage est un contrat social entre familles, où le seul cadet à qui on demande son avis est le garçon. La fille est priée d'obéir. Si elle refuse, maudite ou souvent on la force, il faut respecter l'ordre établi. » Dans cette situation il y a peu de place pour les sentiments. Puisque la fille n'a pas le choix de rester célibataire, elle ne peut pas choisir son mari. 
Au contraire, on demande l'avis du garçon. Il existe aussi d'autres romanciers qui abordent le mariage forcé. Perpétue de Mongo Beti doit quitter l'école pour épouser un homme qu'elle n'aime pas.

Une autre manifestation patriarcale qui est ancré à la dot et au mariage est l'excision. La communauté dans Sous Fer excise leurs filles. La romancière révoque cette coutume à travers l'analyse du personnage de Nana. Après la mutilation, elle a enduré une hémorragie sans cesse. Le roman dépeint les évènements comme suit :

Nana se vidait de son sang. Ni sorcier, ni féticheur n'arrivait à la sauver. Elle fut transportée du vestibule au bourré...les tam-tams s'étaient tus. Les griots ne chantaient plus. Le karignan ne résonnait plus. La vie de Nana était en danger, car elle se trouvait au stade de l'inconscience (Keïta, 2013, p.160).

Nana vide de son sang à cause de la suite de l'excision. Aucun traitement coutumier n'a pu stopper le saignement. Il faut repérer que Fata, la mère de Nana, a fui de l'excision cependant, elle n'a pas pu défendre sa fille contre la mutilation malgré la sensibilisation qu'elle menait contre cette pratique diabolique. La romancière décrit les effets dégoûtants du processus d'excision des filles. L'excision s'est présentée à nous comme un obstacle qu'on doit surmonter et aussi comme un procédé désagréable et douloureux. L'excision est entourée de silences. Quand Nana va à la campagne, elle ignore ce qui l'attend là-bas jusqu'au jour de l'opération. Pourtant, la romancière brise ce silence pour accentuer les problèmes liés à cette pratique. Les vieilles femmes qui font les opérations usent des dispositifs non pasteurisés qui, peuvent transmettre le SIDA et les autres maladies communicables à travers le sang. Les filles sont marginalisées, parce qu'elles ne possèdent pas les procédés pour se défendre auprès ces pratiques atroces et rigides où elles ne boivent pas aucun analgésique afin de contrôler la douleur. À travers cette histoire, la romancière brise le tabou qui existe autour de ce procédé.

L'excision est une coutume risquée aux effets émouvants sur la santé des jeunes filles. Elle est un procédé imbibé dans les us maliens. Le débat de l'excision fait principalement le sujet de châtiments moraux et interpelle toujours aux combats menés par les femmes contre les pratiques antiques. La sexualité et tous les actes anciens autour d'elle sont des outils d'oppositions présentes dans les romans. Normalement en Afrique, l'enfant n'appartient pas à ses parents, pourtant à la société entière. Il arrive que quand les parents déclinent l'excision sur leur fille, un autre membre de la famille peut exciser tout de même l'enfant. Comme on témoigne dans le cas de Nana. Les mères n'ont pas droit de s'opposer à leurs bellesmères. L'excision apporte une double dimension. Elle est traumatique et elle prive la femme $\mathrm{du}$ plaisir sexuel. Toutefois, l'excision transforme négativement la vie des femmes.

Il existe aussi d'autres romans qui traitent le thème de l'excision. Le roman Rebelle (2000) de Fatou Keïta aborde le silence lié à l'excision. Tandis que La voie du salut (1985) d'Aminata Maïga Ka traite ce phénomène comme une tradition protégée. Calixthe Beyala dans Tu t'appelleras Tanga (1990) analyse aussi l'excision.

En Afrique, à partir du jour de son mariage, la femme devient la possession de sa belle-famille, particulièrement après avoir payé la dot. À cause de cette emprise, après la mort de son époux ou à cause de son infertilité, l'épouse est forcée d'épouser le frère ou le cousin de son défunt époux. Le lévirat est une coutume qui subjugue la femme. Il enlève son droit de choisir son époux. Le lévirat est directement attaché au veuvage. Dans Les Mamelles de l'amour, quand Nana perd son mari, elle dit « si le lévirat demeurait une forme d'organisation sociale pour la prise en charge de la veuve et pour sa réinsertion dans sa belle-famille, il ne devrait en aucun cas être imposé à celle qui ne le désirait pas. » Son beau-père qui est cupide et avide lui enlève de tout héritage ce qui augmente sa douleur. Par conséquence, elle finit par délaisser les démarches en justice destinées, toutefois, à la remettre dans ses droits et ceux de son fils comme les vrais héritiers des biens de Kary selon la loi. Nana rencontre beaucoup d'épreuves comme la 
sorcellerie et la maladie. Le résultat est qu'elle tombe dans un coma duquel elle se ranimera deux ans après.

Dans Sous Fer, le narrateur en parle dans l'extrait suivant au sujet du personnage de Kanda : "Selon les coutumes de son village, les femmes de son frère aîné devraient lui revenir un jour si leur mari venait à disparaître...étant le plus âgé des frères il était l'héritier désigné, et il savait que les ressources matérielles, financières et humaines du défunt était considérées comme son héritage » (Keïta, 2013, p.13). Dans cette optique, sa préférence d'une vie monogame a conçu la résistance dans sa famille et des faussetés à cause de son choix imprévu. Sa famille attendait qu'il préfère le choix polygame si par hasard il survenait la nécessité de marier les épouses de ses frères. La romancière veut exposer qu'il y a encore des gens qui encouragent le lévirat bien que les contributions négatives qu'il pourrait emporter. À Mouruba, une place fictive dans Sous Fer, la polygamie « était admise par la coutume et le lévirat demeurait un devoir pour les frères du défunt. C'était une façon pour eux, selon les coutumes, d'aider les veuves à se consoler du décès de leur mari » (Keïta, 2013, p.71). Cette société affirme l'avenir des veuves par le lévirat.

Dans Celles qui attendent de Fatou Diome, la narration expose ce qui suit : « Non seulement Lamine était plus jeune que ses belles-sœurs, mais son bref passage à l'école française lui avait ouvert ses yeux sur un autre mode de vie. La polygamie il n'en voulait pas ; chauffer la couche d'une épouse qu'on n'a pas choisie lui semblait encore plus insupportable » (Diome, 2010, p.15). En invoquant cette scène avec Lamine qui décline de suivre les suppliques de us sociales, l'auteure démontre la potentialité d'abandonner les coutumes funestes à son bonheur. Elle établit également que l'alphabétisation soutient la prise de conscience. De ce fait, Lamine préfère élire son épouse.

Dans Sous Fer, Kanda exprime son regret de son choix d'un mariage monogame : "le régime expressément monogame n'a jamais été apprécié au sein de ma communauté. Ce choix que j'ai fait, ma famille le gardera comme le plus grand camouflet que je lui aie donné » (Keïta, 2013, p.26). Pour avoir enceinté son épouse avant le mariage Kanda se trouve dans une alliance monogame comme châtiment de son beaupère. Pour ce couple, leur désir et leur démarche pour inverser les règles préétablies et l'effort de s'affranchir des mœurs anciennes produirait une raison suffisante pour se faire exiler sans fin par les villageois. L'isolation de ce paire se résume dans les lignes suivantes : " À l'occasion du séjour de son mari à Muruba, aucun cadeau n'avait été suffisamment beau pour Fata pour forcer la sympathie de sa belle-famille...À son arrivée, Kanda fut reçu par les siens avec une froideur digne des Malinkés mécontents et déçus par leur frère " (Keïta, 2013, p.38). Ayant une union monogame contre les attentes de la famille de Kanda, ce couple endure aux mains de la communauté qui met en avant les mariages polygames. Un grand nombre de femmes en Afrique sont affectées par le problème de la polygamie. Même si quelques hommes continuent d'épouser plusieurs femmes, un pourcentage d'eux croit que la polygamie est un enclos. La citation ci-dessus illustre très bien que la société ne favorise pas l'affranchissement d'autrui de mœurs qui l'étouffent. Selon Mbaye (2018, p.195) : «Le fait que l'homme n'avait jamais pris une deuxième épouse suffisait pour l'exposer à la vindicte masculine. » De ces propos, il faut noter que $l^{\prime}$ incapacité d'épouser une deuxième femme peut poser plusieurs problèmes selon la société.

Pour les épouses, la maternité garantit la permanence et la stabilité dans le mariage et dans le clan. Le mauvais désastre qui peut bouleverser une épouse est l'inaptitude d'accoucher. La société africaine n'a pas de place pour une femme stérile. Dans Quand les cauris se taisent le mari de Titi a épousé une seconde femme parce qu'elle ne pouvait pas lui donner un enfant. De ce fait, sa bellefamille avait pressionné son mari pour se remarier. Titi s'est divorcée de lui et elle a trouvé un emploi. À travers ce personnage, la romancière démontre qu'il y a plusieurs 
possibilités pour les femmes même si la société et ses mœurs les confinent dans le foyer. Titi aimait tellement son mari qu'elle avait décroché sa vie professionnelle pour rester dans son foyer. Cependant, cet amour ne pouvait pas remplacer les enfants qu'elle ne pouvait pas lui donner. La vie de Titi démontre qu'il y a les femmes qui ne sont plus reléguées dans les foyers. La romancière présente le mariage comme une bataille continue. Il faut se fait aimer par son mari et par sa belle-famille afin d'éviter qu'il épouse une autre femme. Plus tard, la femme se bat pour demeurer l'épouse préférée quand les coépouses arrivent dans son foyer. Titi dit « quel est ce sadique qui a inventé la polygamie, cette guillotine pour les femmes? D'ailleurs, la polygamie est pire qu'une guillotine. Elle ne tue pas les femmes d'un coup. Elle les tue lentement, à petit feu, faisant durer leur supplice sous le regard rieur et indifférent de la société tout entière. »

Il est vrai que le mariage augmente aux tourments soutenues par ces femmes subjuguées aux séparations et aux mauvais traitements, principalement aux relations conjugales soulignées par l'inhumanité. Traditionnellement, l'accouchement est l'objectif de tout mariage. D'après Amouh (2000, p.29) « dans les sociétés africaines, une femme sans enfant est marginalisée et méprisée. » L'infertilité devient ainsi un fait dramatique. En Afrique, le mariage et la procréation sont étroitement liés et inséparables. On présume presque toujours que la préparation au mariage est la préparation à la procréation. Ceci implique que le mariage est une occasion de contribuer librement, par le biais de la procréation, à la survie du lignage et de la société en général.

Awa (2014, p.18) pose la question suivante : "Quelle nuit peut passer une coépouse lorsqu'elle entend les gémissements et les soupirs de son mari couché avec l'une de ses rivales à quelques mètres de sa propre chambre? " La question précédente anime les inquiétudes des coépouses. Dans la polygamie, les femmes sont considérées comme "la marchandise dont il faut s'accaparer sans pour autant se demander si cette marchandise-là qu'il faut acheter (pot au passe) est douée de raison, de sentiments et d'émotions » (Awa, 2014, p.18). Awa écrit également ce qui suit : « la douleur des femmes! Elles souffrent parce qu'elles sont considérées comme des sous-êtres, du bétail, à qui l'on ne demande que d'être toujours prêtes pour coucher et accoucher » Awa (2014, p.16). Cette mentalité pousse beaucoup d'hommes à la polygamie. Awa (2014) croit que dans le domaine de la conjugalité, le pouvoir masculin s'exercerait au travers des logiques de mariage arrangé. Plus encore, la polygamie est souvent considérée comme un instrument de subordination des femmes et d'expression de la domination masculine. Pour les coépouses, la vie se traduit en concurrence pour le contrôle de l'affection du mari.

\section{CONCLUSION}

Cette étude a fixé comme objectif de traiter la représentation des femmes dans les trois ouvres mentionnés. Dans l'une comme dans l'autre, les femmes s'illustrent par leur bravoure, défiant parfois l'autorité masculine. Nous avons vu que l'héroïsme et la vaillance des femmes forcent un nouveau positionnement social à l'égard de celles-ci. L'objectif était donc de montrer comment elles dénouent les situations inextricables prouvant ainsi aux hommes leur capacité sociale. La domination patriarcale, les pratiques, les croyances culturelles, les lois sur l'héritage sont parmi les problèmes qui atténuent les droits des femmes en Afrique. La plupart des veuves du continent ont subi différentes formes de victimisation de la part de leurs beaux-parents et de la communauté en général. Ces pratiques désagréables ne sont pas seulement traumatiques, mais déprimantes. Ces pratiques sont aussi des exemples de trahison flagrante et de violations des droits fondamentaux des femmes. Le présent document a préconisé donc l'élimination des mœurs qui assujettissent la femme. 


\section{REMERCIEMENTS}

Je remercie mes collègues de l'Université du Zimbabwe pour le soutien dans la rédaction de cet article.

\section{RÉFÉRENCES}

Amouh, F. (2000). Polygamie et conflits missionnaires en Afrique. Thèse de doctorat, littérature comparé, Paris 3.

Awa, T. (1978). La parole aux négresses. Paris : Éditions Denoël.

Bardolph, J. (2002). Études postcoloniales et littérature. Paris : L'Harmattan.

Beauvoir, S. (1976). The second sex. Paris: L'Harmattan.

Beyala, C. (2001). Tu t'appelleras Tanga. Paris : L'Harmattan.

Chassain, A. Clochec, P. Le Meur, C. Lenormand, M et Tregan, M. (2016). Approches expérientielles du fait minoritaire. Revue de Sciences humaines. Tracés 30, 8-10.
Diome, F. (2010). Celles qui attendent. Paris : Flammarion.

Keita, F. (1998). Rebelle. Abidjan: Nouvelles Editions Ivoiriennes.

Keïta, F. (2013). Sous Fer. Paris : L'Harmattan.

Keita, F. (2017). Les mamelles de l'amour. Paris : L'Harmattan.

Keita, F. (2017). Quand les cauris se taisent. Paris : L'Harmattan.

Kesteloot, L. (2001). La percée des femmes dans le roman de mours : Histoire de la littérature Négro-africaine. Paris : Karthala AUF.

Maiga, A. (1985). La voie du salut. Paris: Présence africaine.

Mbaye, A. (2018). Les discours sur l'homosexualité au Sénégal: l'analyse d'une représentationnelle. Berlin : AVM.

Naâmane-Guessous, S. (1988). Au-delà de toute pudeur. La sexualité féminine au Maroc. Casablanca : Eddif.

Zénith, M. (2000). La sexualité. Paris : Heinemann. 